PHYSICAL REVIEW FLUIDS 1, 050506 (2016)

\title{
Placing Marangoni instabilities under arrest
}

\author{
M. Saad Bhamla ${ }^{1, *}$ and Gerald G. Fuller ${ }^{2}$ \\ ${ }^{1}$ Department of Bioengineering, Stanford University, Stanford, California 94305, USA \\ ${ }^{2}$ Department of Chemical Engineering, Stanford University, Stanford, California 94305, USA
}

(Received 9 August 2016; published 12 September 2016)

\begin{abstract}
This paper is associated with a video winner of a 2015 APS/DFD Milton van Dyke Award for work presented at the DFD Gallery of Fluid Motion. The original video is available from the Gallery of Fluid Motion, http://dx.doi.org/10.1103/APS.DFD.2015.GFM.V0040
\end{abstract}

DOI: 10.1103/PhysRevFluids.1.050506

Soap bubbles occupy the rare position of delighting and fascinating both young children and scientific minds alike. Newton [1], Plateau [2], Marangoni [3], and de Gennes et al. [4], not to mention countless others [5], have discovered remarkable results in optics, molecular forces, and fluid dynamics from investigating this seemingly simple system. We present here a compilation of curiosity-driven experiments that systematically investigate the surface flows on a rising soap bubble. From childhood experience, we are familiar with the vibrant colors and mesmerizing display of chaotic flows on the surface of a soap bubble. These flows arise due to surface tension gradients, also known as Marangoni flows or instabilities. In Fig. 1, we show the surprising effect of layering multiple instabilities on top of each other, highlighting that unexpected new phenomena are still waiting to be discovered, even in the simple soap bubble.

We conduct the experiment as follows. We place a plastic chamber containing surfactant solution [0.3 mM sodium dodecyl sulfate (SDS) in water] onto a motorized stage. In this solution, we generate an air bubble ( $1 \mathrm{~mm}$ diameter) at the tip of a U-shaped glass capillary (Fig. 2). We initially position the bubble just beneath the surface. Next, using the stage, we elevate the bubble in controlled, discrete steps to expose a spherical cap of the bubble at the surface. This protruding cap is essentially a draining curved film, which under white light illumination exhibits interference patterns that we record using a color charge-coupled device (CCD) camera.

We find that the Marangoni instabilities can be reproduced by a broad range of soluble surfactants that have negligible surface shear viscosity. Thus, this experiment serves as a facile technique for probing the surface mobility of surfactant systems [6], including artificial lung surfactant therapeutics [7]. However, a complete theoretical analysis of this problem remains an open challenge.

*Corresponding author: bhamla@stanford.edu

Published by the American Physical Society under the terms of the Creative Commons Attribution 3.0 License. Further distribution of this work must maintain attribution to the author(s) and the published article's title, journal citation, and DOI. 


\section{SAAD BHAMLA AND GERALD G. FULLER}

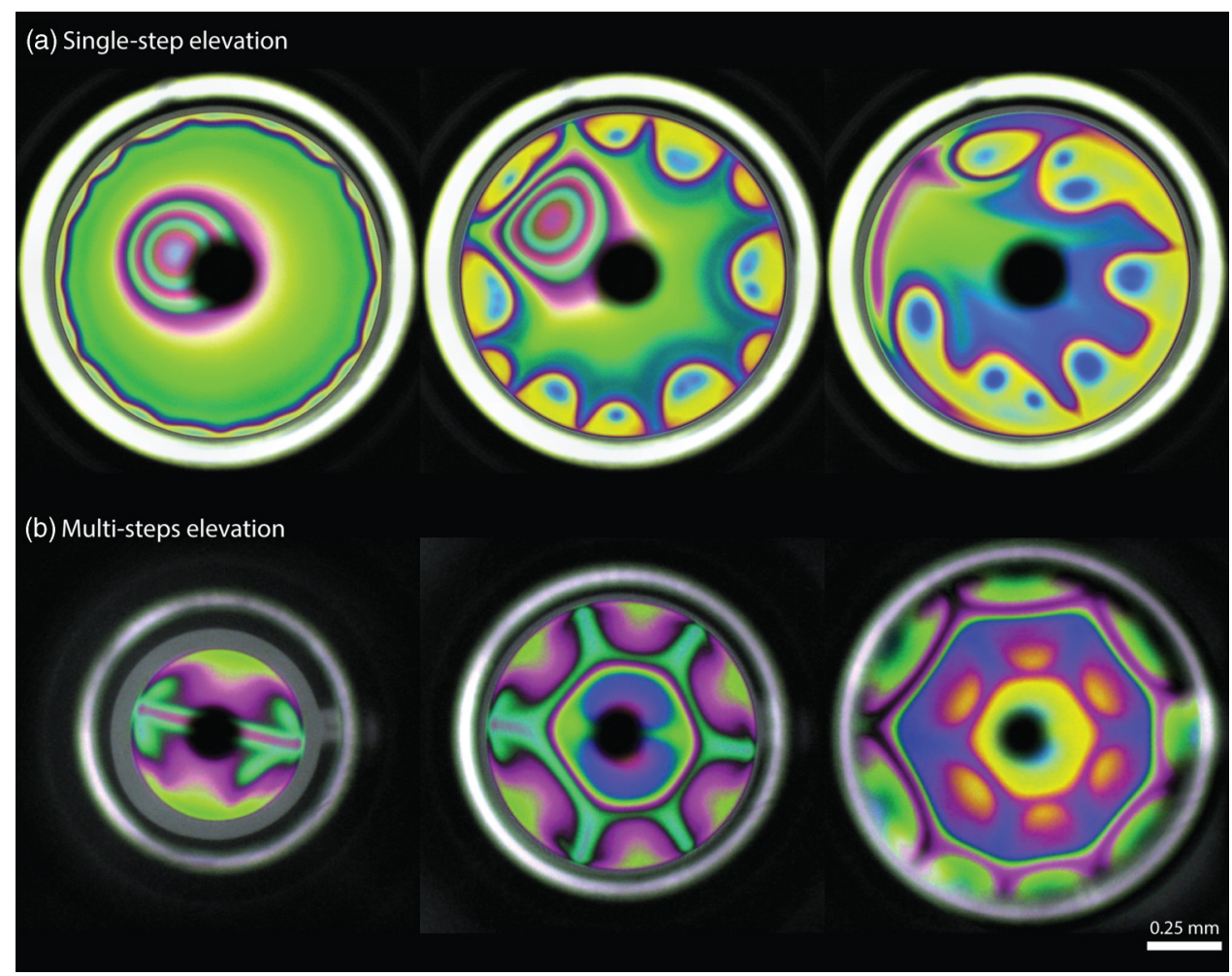

FIG. 1. Snapshots of Marangoni instabilities on a soap film elevated in (a) a single step (0.9 mm) and (b) multiple steps ( 3 steps of $0.3 \mathrm{~mm}$ ). In the first row, the dimple in the center is unstable and flees towards the periphery as plumes of surfactant rise from the periphery, resulting in chaos. However, in the second row, elevation in smaller steps creates a cascade of instabilities that arrest each other, resulting in beautiful dynamic structures. To fully appreciate these incredible flow patterns, we urge the reader to watch the accompanying multimedia video appended as Supplemental Material and located here http://dx.doi.org/10.1103/APS.DFD.2015.GFM.V0040

(a)
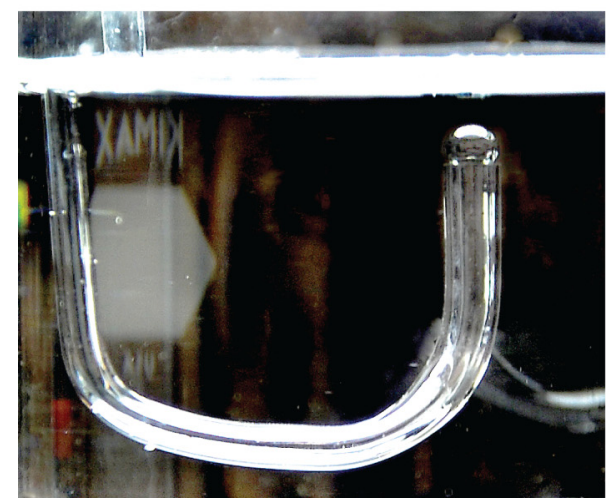

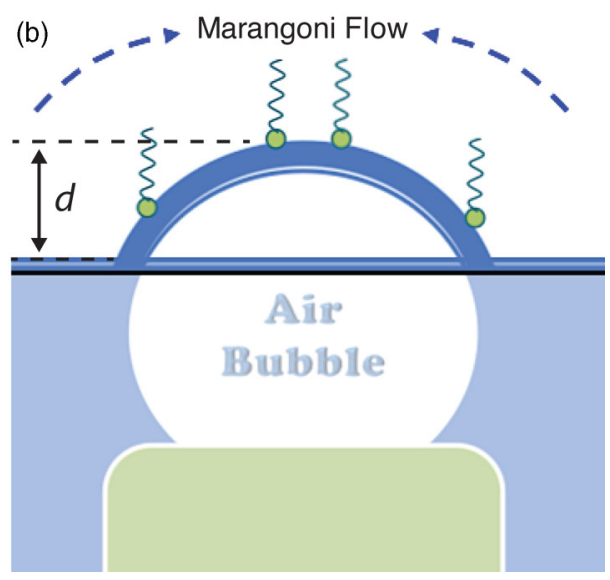

FIG. 2. (a) Image of experimental setup. (b) Schematic illustrating the Marangoni flow generated due to redistribution of surfactant molecules as the bubble is elevated through the solution interface, where $d=0.9 \mathrm{~mm}$ is the vertical displacement from initial starting point just beneath the air-solution interface. 


\section{PLACING MARANGONI INSTABILITIES UNDER ARREST}

We graciously acknowledge Marco A. Àlvarez-Valenzuela and John Frostad for technical support and Yevgenya Strakovsky for video editing.

[1] I. Newton, Opticks, or, a Treatise of the Reflections, Refractions, Inflections, and Colours of Light (Dover Publications, Inc., New York, 1979).

[2] J. Plateau, Experimental and Theoretical Statics of Liquids Subject to Molecular Forces Only (GauthierVillars, Paris, 1873).

[3] C. Isenberg, The Science of Soap Films and Soap Bubbles (Dover Publications, Inc., New York, 1992).

[4] P.-G. De Gennes, F. Brochard-Wyart, and D. Quéré, Capillarity and Wetting Phenomena: Drops, Bubbles, Pearls, Waves (Springer-Verlag, New York, 2004).

[5] C. V. Boys, Soap Bubbles, Their Colours, and the Forces Which Mold Them (Dover Publications, Inc., New York, 1958).

[6] M. S. Bhamla, C. Chai, M. A. Álvarez-Valenzuela, J. Tajuelo, and G. G. Fuller, Interfacial mechanisms for stability of surfactant-laden films, arXiv:1608.02180 (unpublished).

[7] E. Hermans, M. S. Bhamla, P. Kao, G. G. Fuller, and J. Vermant, Lung surfactants and different contributions to thin film stability, Soft Matter 11, 8048 (2015). 